\title{
Analysing the Effect of Platform and Operating System Features on Predicting Consumers' Purchase Intent Using Machine Learning Algorithms
}

\author{
RAMAZAN ESMELI ${ }^{1}{ }^{[}{ }^{\mathrm{a}}$, ALAA MOHASSEB $^{1}{ }^{1}{ }^{\mathrm{b}}$ and MOHAMED BADER-El-DEN ${ }^{1} \mathbb{C}^{\mathrm{c}}$ \\ ${ }^{1}$ School of Computing, University of Portsmouth, Portsmouth, UK \\ \{ramazan.esmeli, alaa.mohasseb, mohamed.bader\}@port.ac.uk
}

\begin{abstract}
Keywords: Purchase intention prediction, Purchase behaviour prediction, browsing behaviour, Classification, Machine Learning

Abstract: Predicting future consumer browsing and purchase behaviour has become crucial to many marketing platforms. Consumer purchase intention is one of the main inputs used as a measurement for consumer demand for new products. In addition, identifying consumers' purchase intent play an important role in recommender systems. In this paper, the effect of using different platforms on users' behaviours is explored. In addition, the effect of users' platforms and their purchase intentions behaviours are investigated. We conduct computational experiments using different machine learning algorithms in order to investigate the using users' operating system and platform types as features. The results showed that the users' purchase intentions and behaviours are correlated with these features.
\end{abstract}

\section{INTRODUCTION}

Predicting future consumer browsing and purchase behaviour has become crucial to many marking platforms. Knowing customers need plays an important role in identifying potential customers (Christy et al., 2018). Moreover, prior information and expert knowledge is an important aspect to obtain reliable and consistent solutions (Mohasseb et al., 2019).

Consumer purchase intention is one of the main inputs that marketing managers use to forecast future sales and to determine how the actions they take will impact consumers' purchasing behaviour (Morwitz et al., 2014),(Esmeli et al., 2020). In addition, these intentions are used as a measurement for consumer demand of new products, in which marketing managers could use as an in indicator of future demand for their products, and to assess how their marketing actions will impact that future.

Furthermore, identifying consumers' purchase intent play an important role in recommender systems when determining which users will be receptive to specific product recommendations (Korpusik et al., 2016), (Esmeli et al., 2019).

\footnotetext{
a (iD) https://orcid.org/0000-0002-2634-6224

b (iD) https://orcid.org/0000-0003-2671-2199

c (iD) https://orcid.org/0000-0002-1123-6823
}

The most common approach taken by many recent studies is to identify the next buyer or the buyer intent using machine learning algorithms such as deep learning (Salehinejad and Rahnamayan, 2016), (Korpusik et al., 2016), other works such as (Kim et al., 2003) used multiple classifier while authors in (Qiu et al., 2015) and (Raphaeli et al., 2017) used unsupervised learning such as association rules. Moreover, consumers buying behaviour is analysed using different methods such as statistical methods (Gupta and Pathak, 2014) and hidden Markov model (Norouzi and Alizadeh, 2016) to identify and recommend a given product or service (Martínez et al., 2020), (Raphaeli et al., 2017), (Bang et al., 2013) (Wang et al., 2015), (Kaatz et al., 2019) and (Liu et al., 2019).

In this paper, the effect of using different platforms and operating systems that users browse websites on users' behaviours, is explored. In order to achieve this aim, the following objectives are defined:

1. Analysing customers buying behaviour and their intent when using different operating systems.

2. Creating a framework to analyse the purchase prediction performance when different operating systems and platform types are utilised as features.

3. Investigating customers buying behaviour when different machine learning algorithms are used.

4. Evaluating the performance of different machine 
learning algorithms on the prediction and the classification of customers intent when using platform types and operating systems users are using to connect to different websites.

The paper is organised as follows: In Section 2, we discuss related work on consumer behaviour analysing and purchase prediction. Section 3 describes the details of the datasets used in our proposed method. In Section 4, we give experimental results and discuss the performance of the proposed methods. Finally, in Section 5, we conclude the paper and give a new research direction.

\section{RELATED WORKS}

In this section, we review the existing literature on consumer purchase prediction using different methods. Recent studies used machine learning algorithms. In (Kim et al., 2003), authors proposed a Genetic Algorithm (GA)-based multiple classifiers combining method for the prediction of the customers' purchase behaviour. The proposed approach combines individual decisions by multiple classifiers. The experiment for a case from a leading Ecommerce company in Korea showed that the proposed combined method outperforms any individual classifiers. Furthermore, two experiments were conducted. The first experiment showed that the proposed algorithm could improve the prediction accuracy of the purchase propensity and the second experiment, showed that this method has better performance than other combining methods. Works in (Korpusik et al., 2016) used deep learning techniques for predicting customer purchase behaviour from Twitter data that recommender systems could leverage. Authors collected a labelled corpus of buy/not buy users and their tweets. A neural network-based classifier performed best at predicting whether a tweet is relevant to purchase behaviour, with an accuracy of $81.2 \%$ on mobile devices and $80.4 \%$ on cameras. Results showed that the use of a deep learning model that incorporates sequential information performed better than ignoring sequential information for the purchase prediction task. In (Gupta and Pathak, 2014) authors proposed a framework which combined different techniques of machine learning, data mining and statistical methods to predict the purchase behaviour of an online customer by selecting an appropriate price range based on dynamic pricing. The proposed framework has been tested on a large dataset for an e-commerce firm. While (Salehinejad and Rahnamayan, 2016) proposed a customer behaviour prediction model using recurrent neural networks (RNNs) based on the client loy- alty number (CLN), recency, frequency, and monetary (RFM) variables. The experiment results showed that RNNs could predict RFM values of customers efficiently. This model can be later used in recommender systems for exclusive promotional offers and loyalty programs management.

Moreover, other studies used unsupervised learning, such as association rules. Authors in (Qiu et al., 2015) proposed a predictive framework for customer purchase behaviour in the e-commerce context called CustOmer purchase pREdiction modeL (COREL). Associations among products were investigated and exploited to predicate customer's motivations, and customer preferences for product features were learned and subsequently used to identify the candidate products most likely to be purchased. In addition, in this study, the authors investigated three categories of product features and develop methods to detect customer preferences for each of these three categories. Experiments conducted on a real dataset showed that customer preference for particular product features plays a key role in decision-making and that COREL greatly outperforms the baseline methods. While in (Raphaeli et al., 2017) authors used clickstream to compare browsing behaviour in mobile and PC sessions. Analysis has been conducted to identify the differences in site usage characteristics across different channels, in which the analysis showed that user engagement in pc session is higher than mobile sessions. In addition, sequential association rule mining was applied to the clickstream data navigation patterns, which represent formal combinations of web page transitions. The final analysis demonstrated that browsing behaviour tends to be more task-oriented in mobile sessions compared to PC sessions.

Furthermore, consumers buying behaviour is analysed using different methods such as graphical probability model. Works in (Wen et al., 2018) proposed a graphical probability model that exploits the payment data to discover customer purchase behaviour in the spatial, temporal, payment amount and product category aspects, named STPC-PGM. As a result, the mobility behaviour of an individual user showed that it could be used to predict with a probabilistic graphical model that accounts for all aspects of each customer's relationship with the payment platform. In order to achieve real-time advertising, an online framework was developed that efficiently computes the prediction results. The experiment results showed that STPC-PGM is effective in discovering customers' profiling features, and outperforms the state-of-theart methods in purchase behaviour prediction. While in (Norouzi and Alizadeh, 2016), a hidden Markov 
model was used, in which authors proposed a hybrid method based on hidden Markov model and multinomial choice model to analyse customer choice behaviour. The proposed method was applied to the retail store data set.

In addition, authors in (Martínez et al., 2020) developed advanced analytics tools that predict future customer behaviour in the non-contractual setting. A dynamic and data-driven framework was built for predicting whether a customer is going to make a purchase at the company within a certain time frame in the near future. For that purpose, the authors proposed a new set of customer-relevant features that derive from times and values of previous purchases. These customer features are updated every month, and state-of-the-art machine learning algorithms are applied for purchase prediction. Using a data set containing more than 10,000 customers and a total number of 200,000 purchases an accuracy score of 89\% was obtained and an Area Under Curve(AUC) value of 0.95 for predicting next month purchases on the test data set. (Choudhury and Nur, 2019) proposed an engineered approach to classifying potential customer based on previously recorded purchase behaviour. Machine learning algorithms were applied to find a pattern and predict potential customers by using the classification results as ground truth. The proposed approach achieved an accuracy of $99.4 \%$.

Authors in (Bang et al., 2013) investigated consumer browsing and purchase behaviour. The result showed that the frequency and purchase time irregularity was found to have a positive impact on mobile commerce adoption. The results also suggested that search cost influences the decision to adopt mobile commerce. In addition, the consumers who search multi-item or categories at a time, engaged in active search, and conducted a thorough search, are less likely to adopt mobile-commerce. Authors in (Wang et al., 2015) used a dataset from an internet-based grocery retailer to evaluate the changes in customers' spending behaviour when M-shopping is adapted, such as using smartphones, tablets and online. Results showed that the number of orders placed per year increased as customers adopt M-shopping. In (Carreón et al., 2019), the effect of exposing time to product advertisement on TV is investigated on users' purchase behaviours. They used machine learning models in order to analyse the influence of purchase behaviour from the advertisement. Results show that the effect of advertisement on TV has a minimal influence factor on users' purchase behaviours.

While, in (Kaatz et al., 2019), the different impacts of different devices were examined, such as desktop computers, tablets, and smartphones on the success of various marketing channels dependent on the device. This study demonstrated that desktop users should be approached by marketing channels which induce casual browsing behaviour (e.g., newsletter, social media, referrer, SEO). In contrast, mobile users are more likely to use marketing channels that do not require an extended information search, such as SEA or direct visits to familiar stores. In addition, customer experience is important in improving attribution outcomes (e.g., conversion rates) by combining clickstream and survey data to understand consumers' decision processes. Finally, in (Liu et al., 2019) authors explored whether consumers' app adoption stimulates additional purchases and how this change in purchase behaviour differs across customers with different levels of spending share for different product categories and customer loyalty. Transactional data from a Chinese online retailer were used. Results showed that app adopters have higher purchase incidence, buy more frequently, and spend more in each order than non-adopters. Furthermore, results suggest that apps are worth investing in despite their similarity to mobile websites and can induce nonloyal customers to purchase more and thus potentially foster these customers' loyalty.

\section{DATASET ANALYSIS}

In order to explore the effect of using different platforms on users' behaviours, six datasets were used provided by a UK based content personalisation company. The dataset statistics are shown in Table 1 give information about the number of interactions, the number of unique items and users. Also, the datasets are analysed in terms of the statistics about which platforms and devices e-shoppers are using to visit the six e-commerce platforms.

Table 1: The statistics about the number of the items, users and interactions in the dataset

\begin{tabular}{lccc}
\hline Dataset & \#user & \#item & \#interactions \\
\hline dataset 1 & 148832 & 3835 & 551851 \\
dataset 2 & 361330 & 33273 & 768999 \\
dataset 3 & 478089 & 20480 & 456077 \\
dataset 4 & 301089 & 9111 & 789059 \\
dataset 5 & 215240 & 17431 & 519251 \\
dataset 6 & 266871 & 34152 & 800985 \\
\hline
\end{tabular}

Table 2 shows the the number of operating systems that users use to connect to each website. It can be seen in Table 2 that in most websites IOS os has superiority over others except dataset 1 in which users 
mostly use Windows os to connect to websites. Moreover, we eliminated operating systems which have less than 100 connections to the websites. The operating systems which have been eliminated are blackberry, Fedora, Debian, FreeBSD, OpenBSD, Windows 2000, Windows Phone, Windows RT, Firefox OS and Ubuntu.

Table 3 shows the number of interactions comes from each platform. Except Dataset 1, in all other datasets the number of mobile users has more than the number of users from other platforms. Furthermore, We analyse the dataset in terms of the distribution of interaction and its outcome over weekday, day of the month, operating systems and platforms. We select only Dataset 6 since it has the highest number of the interactions, also we have limited space to put other datasets' analysis.

Figure 1 shows the number of interactions over the day of the week by the outcome of the interaction for dataset 6. This Figure shows that Wednesday has the most number of interactions among the other day of the week.

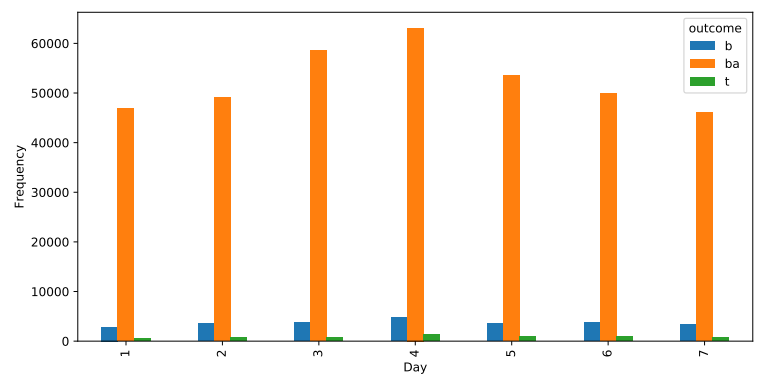

Figure 1: User-Website interaction distribution over the days of the week by outcome of the sessions for Dataset 6

Figure 2 shows the frequency of the interactions on the hours of the day by outcome for dataset 6 . It shows that users mostly active after midday.

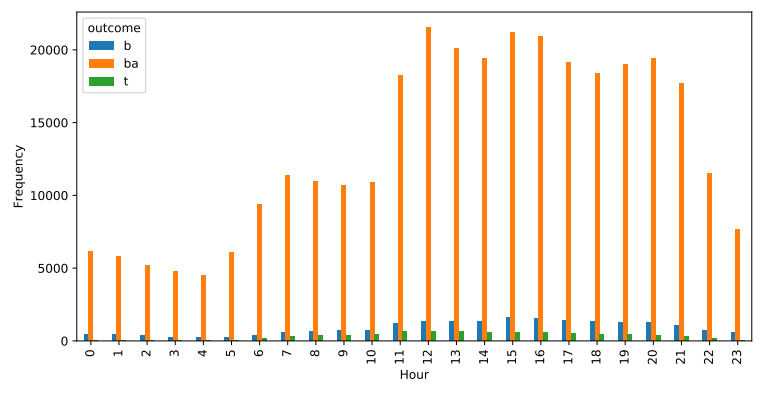

Figure 2: User-Website interaction distribution over the hours of the day by outcome of the sessions for Dataset 6
We also analysed the session outcome and userwebsite interactions distribution for each operating system for dataset 6 . As seen in Figure 3, website visitors mostly use IOS operating system

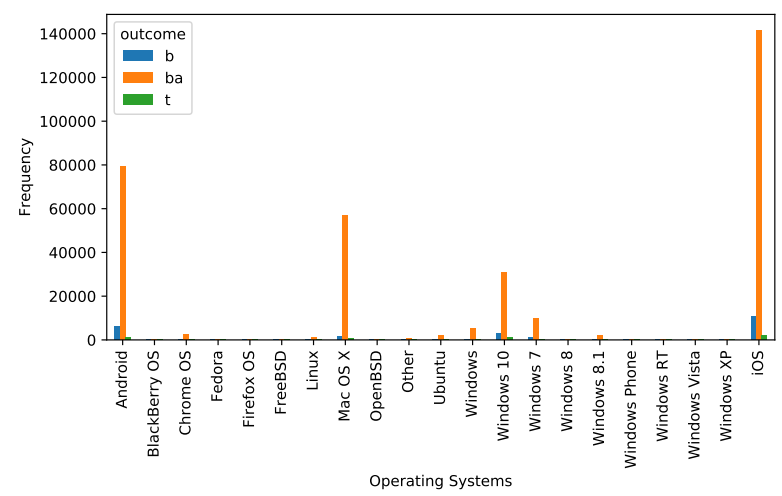

Figure 3: Distribution of User-Website interaction for the operating systems grouped by outcome of the sessions for Dataset 6

Finally, we analyse the dataset in terms of the number of user interactions and platform types (Figure 4). As seen in Figure 4, most of the visitors use a mobile platform.

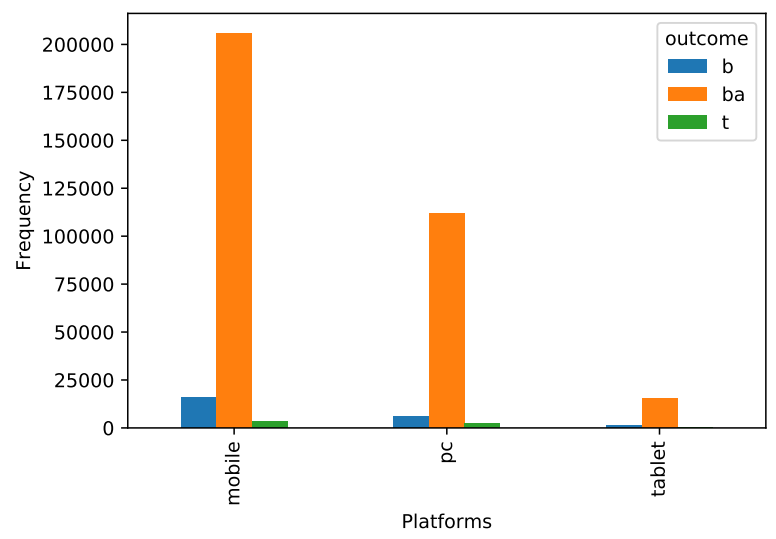

Figure 4: Distribution of User-Website interaction for the platforms grouped by outcome of the sessions for Dataset 6

\section{Experiments, Results and Discussion}

In this section we give the details of the experiments and results. Figure 5 shows the brief overview of the way we follow for the experimental evaluation of the proposed approach.

Three well-known machine learning prediction models are used to explore the effect of users' platforms on their purchase intention behaviours. These 
Table 2: Statistics about Operating Systems Users Use to Visit the Websites

\begin{tabular}{lllllll}
\hline Operating Systems & Dataset 1 & Dataset 2 & Dataset 3 & Dataset 4 & Dataset 5 & Dataset 6 \\
\hline Android & 7452 & 97126 & 217499 & 228532 & 195786 & 95151 \\
Chrome OS & 218 & 658 & 8002 & 3718 & 1609 & 3234 \\
Linux & 28456 & 2446 & 2189 & 864 & 19723 & 2008 \\
Mac OS X & 14250 & 47493 & 96197 & 57929 & 83075 & 95662 \\
Other & 31719 & 77735 & 2483 & 24025 & 19764 & 18437 \\
Windows & 21692 & 4888 & 7682 & 1976 & 6065 & 16312 \\
Windows 10 & 141310 & 106667 & 103470 & 109707 & 163579 & 131327 \\
Windows 7 & 66234 & 58595 & 28607 & 22115 & 87893 & 30549 \\
Windows 8 & 674 & 343 & 560 & 523 & 484 & 315 \\
Windows 8.1 & 10953 & 3073 & 6435 & 5613 & 54067 & 5394 \\
Windows XP & 911 & 272 & 500 & 443 & 874 & 270 \\
iOS & 12298 & 475057 & 370670 & 431394 & 210895 & 450333 \\
\hline
\end{tabular}

Table 3: Statistics about the Platforms users use to connect to websites

\begin{tabular}{llll}
\hline Dataset & Mobile & Pc & Tablet \\
\hline Dataset 1 & 16191 & 317286 & 3562 \\
Dataset 2 & 522675 & 303614 & 48467 \\
Dataset 3 & 542152 & 259108 & 45921 \\
Dataset 4 & 530621 & 229191 & 128246 \\
Dataset 5 & 438576 & 304657 & 102158 \\
Dataset 6 & 307616 & 298133 & 244454 \\
\hline
\end{tabular}

prediction models are K-Nearest Neighbour (KNN), Decision Tree (DT), and Bagging classifier. The reason of selecting three different classifiers is that they have different ways of model learning. For example, Bagging classifier is a type of ensemble learning models (Brzezinski et al., 2018),(Bader-El-Den et al., 2018). Furthermore, in order to validate the proposed approach and evaluate the ability of the machine learning prediction models, we run experiments with ten cross-validations. The F-score was used as the metric to measure the performance of the models. F-score reflects both precision and recall metrics (Zhu et al., 2019) and common metric in order to validate the performance of prediction models.

\subsection{Feature Engineering}

In order to train the classification models the following features were selected:

1. Day of the Week: This feature indicates the day of the week the user interacted with the platform. This feature has an important indication of users' purchase behaviour since most of the users prefer to buy products on the weekdays

2. Hour of Day: Shows the hour that a user has browsed the products. Statistical analysis shows that users mostly buy products after working hours.

3. Session Duration: Shows how many minutes a session lasted.

4. Longitude: Longitude parameter of the location that a user connected from to a website.

5. Latitude: Latitude parameter of the location that a user connected from to a website.

6. number of previous purchases: shows the number of the products a user purchased in the previous sessions.

7. number of previous sessions: indicates the number of times a user has visited the website.

8. number of pages viewed: shows the number of the viewed pages in the session.

We have added operating systems and the platform types as features to analyse their effect on the users' purchase behaviour prediction.

1. operating systems (os): shows which operating system is used to browse the website.

2. platform types (ff): shows which platform type (mobile, tablet, pc) a user is using to browse the website.

\subsection{Results}

In this section, the results of the machine learning algorithms are presented and analysed for each of the six datasets. Table 4 shows the overview of the results for all of the datasets. In the following sections, the results will be discussed in more details for each dataset. 

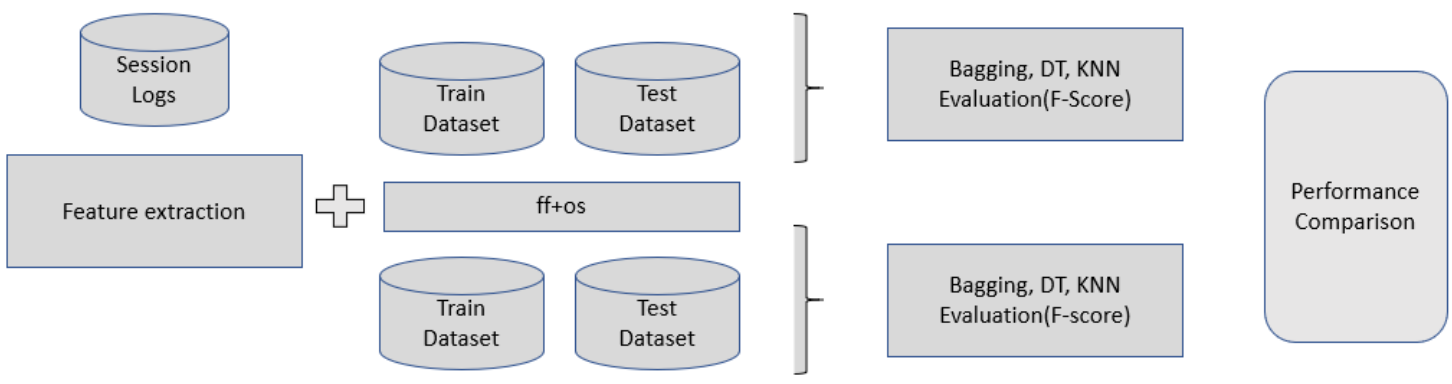

Figure 5: Experimental design to analyse the effect of using operating systems and platform types as features

Table 4: Performance of the classifiers (K-Nearest Neighbour (KNN), Decision Tree (DT) and Bagging with (+) and without (-) using operating systems (os) and platform types (ff) as features.

\begin{tabular}{|c|c|c|c|c|c|c|c|c|c|c|c|c|}
\hline & \multicolumn{2}{|c|}{ Dataset 1} & \multicolumn{2}{|c|}{ Dataset 2} & \multicolumn{2}{|c|}{ Dataset 3} & \multicolumn{2}{|c|}{ Dataset 4} & \multicolumn{2}{|c|}{ Dataset 5} & \multicolumn{2}{|c|}{ Dataset 6} \\
\hline ML & $+o s, f f$ & - os, ff & $+o s, f f$ & $-o s$, ff & $+o s, f f$ & - os, ff & $+o s$, ff & $-o s$, ff & $+o s, f f$ & $-\mathbf{o s}, \mathbf{f f}$ & $+o s, f f$ & $-\mathrm{os}, \mathrm{ff}$ \\
\hline DT & 0.593 & 0.588 & 0.838 & 0.835 & 0.709 & 0.699 & 0.828 & 0.820 & 0.816 & 0.806 & 0.857 & 0.852 \\
\hline Bagging & 0.581 & 0.574 & 0.850 & 0.845 & 0.699 & 0.686 & 0.843 & 0.832 & 0.837 & 0.820 & 0.874 & 0.866 \\
\hline KNN & 0.395 & 0.394 & 0.693 & 0.694 & 0.564 & 0.563 & 0.631 & 0.634 & 0.643 & 0.644 & 0.731 & 0.730 \\
\hline
\end{tabular}

\subsubsection{Dataset 1}

When comparing the performance of DT, Bagging, and KNN, DT has a better F-score when the operating systems (os) and platform types (ff) features are used. While KNN classifier has performed very similar when these attributes are included and excluded. In addition, KNN classifier shows the worst F-score score among the other classifiers. Moreover, interestingly, DT classifier has performed better than Bagging. Also, prediction models have the worst F-score comparing to the other datasets in all classification models (DT:59.3 \%, Bagging: 58.1 \%, and KNN 39.5 $\%)$. Overall, using os and ff types as features has improved the F-score of classification models.

\subsubsection{Dataset 2}

In Dataset 2, Bagging classifier has the best F-score, followed by DT with $85 \%$ and $83.8 \%$ respectively. While KNN classifier has the worst F-score. In this Dataset, classification results show that ensemble classifier has better performance in predicting users' purchase intention. Also, adding the os and ff types as features helped to get a better prediction score when DT and Bagging classifiers were used.

\subsubsection{Dataset 3}

Dataset 3 performance results show the similarity with the performance results of Dataset 1 in terms of how the prediction models are performed. When os and $\mathrm{ff}$ were used as features, all the classifiers per- formed better. However, KNN classifier showed almost similar performance when os and ff used.

\subsubsection{Dataset 4}

Bagging classifier outperformed the other classifiers with an $84.3 \%$ when os and ff features are added. Interestingly, we could not see any improvement on KNN classifier, while Bagging and DT show better F-score when os and ff are included.

\subsubsection{Dataset 5}

Similarly, in Dataset 4, adding os and ff did not help to improve the performance of the KNN classifier, while Bagging gives the best F-score with $84.3 \%$ when os and $\mathrm{ff}$ are included as features.

\subsubsection{Dataset 6}

Classifier models produced the highest performance scores in this dataset comparing to other datasets. Also, including os and ff as features helped to get better performance in all classifiers. In addition, Bagging has the best F-score, while $\mathrm{KNN}$ is the lowest, with $87.4 \%$ and $73.1 \%$ respectively.

\subsubsection{Overall Result Analysis}

DT and Bagging show the superiority over the KNN classifier on all datasets for purchase prediction. On the other hand, using os and ff as features does not have the same effect as DT and Bagging for each dataset. Generally, os and ff have an indication role 
over users' purchase intention and using these features is useful to determine purchase prediction. Interestingly, KNN classifier did not show the positive effect when os and ff are added as features to train the classification models on datasets 2,4 and 5 .

\subsection{Discussion}

The effects of adding new features that show the users' behaviours are investigated on performance improvement for purchase intention prediction (Mokryn et al., 2019) (Carreón et al., 2019). Authors in (Mokryn et al., 2019) investigated the feature of product trendiness on purchase prediction performance. They found a significant difference when the product trendiness was included. In addition, works in (Wang et al., 2015) analysed the correlation between devices that are connected to the users when visiting an e-commerce website and their purchase behaviours. They found that there is a positive correlation in their buying behaviour and the usage of mobile devices to visit e-commerce websites. However, they did not investigate the effect of using mobile devices on purchase prediction using machine learning algorithms. In our research, we conducted an experimental analysis in order to analyse the effect of operating systems and platform types on the prediction of users' purchase intention using machine learning algorithms. The results showed that similar to considering product trendiness as feature (Mokryn et al., 2019); there is a positive correlation on purchase prediction performance in the sessions when the machine learning algorithms are aware of users' operating systems and platform types. The limitation of this work is that we compared the difference of the machine learning algorithms' performance on purchase prediction before and after adding both features however one of the features(os or ff) could lead better correlation result.

\section{Conclusion and Future works}

In this work, we investigated the effect of the operating system and platform types that users use to browse the e-commerce platforms on their purchase behaviour. In order to identify their impact, we run computational experiments with and without including operating system and platform types as features. The results showed that the users' purchase behaviours are correlated with these features.

As future research, we will integrate the purchase prediction results with session-based recommender systems in order to improve personalisation when users browse the products in a session.

\section{REFERENCES}

Bader-El-Den, M., Teitei, E., and Perry, T. (2018). Biased random forest for dealing with the class imbalance problem. IEEE transactions on neural networks and learning systems, 30(7):21632172.

Bang, Y., Han, K., Animesh, A., and Hwang, M. (2013). From online to mobile: Linking consumers' online purchase behaviors with mobile commerce adoption. In PACIS, page 128.

Brzezinski, D., Stefanowski, J., Susmaga, R., and Szczech, I. (2018). Visual-based analysis of classification measures and their properties for class imbalanced problems. Information Sciences, 462:242-261.

Carreón, E. C. A., Nonaka, H., Hentona, A., and Yamashiro, H. (2019). Measuring the influence of mere exposure effect of tv commercial adverts on purchase behavior based on machine learning prediction models. Information Processing \& Management, 56(4):1339-1355.

Choudhury, A. M. and Nur, K. (2019). A machine learning approach to identify potential customer based on purchase behavior. In 2019 International Conference on Robotics, Electrical and Signal Processing Techniques (ICREST), pages 242-247. IEEE.

Christy, A. J., Umamakeswari, A., Priyatharsini, L., and Neyaa, A. (2018). Rfm ranking-an effective approach to customer segmentation. Journal of King Saud University-Computer and Information Sciences.

Esmeli, R., Bader-El-Den, M., and Abdullahi, H. (2020). Using word2vec recommendation for improved purchase prediction. In IEEE World Congress on Computational Intelligence (WCCI) 2020. Institute of Electrical and Electronics Engineers.

Esmeli, R., Bader-El-Den, M., and Mohasseb, A. (2019). Context and short term user intention aware hybrid session based recommendation system. In 2019 IEEE International Symposium on INnovations in Intelligent SysTems and Applications (INISTA), pages 1-6. IEEE.

Gupta, R. and Pathak, C. (2014). A machine learning framework for predicting purchase by online customers based on dynamic pricing. Procedia Computer Science, 36:599-605. 
Kaatz, C., Brock, C., and Figura, L. (2019). Are you still online or are you already mobile?predicting the path to successful conversions across different devices. Journal of Retailing and Consumer Services, 50:10-21.

Kim, E., Kim, W., and Lee, Y. (2003). Combination of multiple classifiers for the customer's purchase behavior prediction. Decision Support Systems, 34(2):167-175.

Korpusik, M., Sakaki, S., Chen, F., and Chen, Y.-Y. (2016). Recurrent neural networks for customer purchase prediction on twitter. In CBRecSys@ RecSys, pages 47-50.

Liu, H., Lobschat, L., Verhoef, P. C., and Zhao, H. (2019). App adoption: The effect on purchasing of customers who have used a mobile website previously. Journal of Interactive Marketing, 47:16-34.

Martínez, A., Schmuck, C., Pereverzyev Jr, S., Pirker, C., and Haltmeier, M. (2020). A machine learning framework for customer purchase prediction in the non-contractual setting. European Journal of Operational Research, 281(3):588-596.

Mohasseb, A., Bader-El-Den, M., and Cocea, M. (2019). A customised grammar framework for query classification. Expert Systems with Applications, 135:164-180.

Mokryn, O., Bogina, V., and Kuflik, T. (2019). Will this session end with a purchase? inferring current purchase intent of anonymous visitors. Electronic Commerce Research And Applications, 34:100836.

Morwitz, V. et al. (2014). Consumers' purchase intentions and their behavior. Foundations and Trends ${ }^{\circledR}$ in Marketing, 7(3):181-230.

Norouzi, P. and Alizadeh, S. H. (2016). An extension of multinomial choice model for customer purchase behavior analysis. In 2016 Artificial Intelligence and Robotics (IRANOPEN), pages 6166. IEEE.

Qiu, J., Lin, Z., and Li, Y. (2015). Predicting customer purchase behavior in the e-commerce context. Electronic commerce research, 15(4):427452.

Raphaeli, O., Goldstein, A., and Fink, L. (2017). Analyzing online consumer behavior in mobile and pc devices: A novel web usage mining approach. Electronic commerce research and applications, 26:1-12.

Salehinejad, H. and Rahnamayan, S. (2016). Customer shopping pattern prediction: A recurrent neural network approach. In 2016 IEEE Sym- posium Series on Computational Intelligence (SSCI), pages 1-6. IEEE.

Wang, R. J.-H., Malthouse, E. C., and Krishnamurthi, L. (2015). On the go: How mobile shopping affects customer purchase behavior. Journal of Retailing, 91(2):217-234.

Wen, Y.-T., Yeh, P.-W., Tsai, T.-H., Peng, W.-C., and Shuai, H.-H. (2018). Customer purchase behavior prediction from payment datasets. In Proceedings of the Eleventh ACM International Conference on Web Search and Data Mining, pages 628-636.

Zhu, G., Wu, Z., Wang, Y., Cao, S., and Cao, J. (2019). Online purchase decisions for tourism ecommerce. Electronic Commerce Research and Applications, 38:100887. 